\title{
Study of microhardness and electrical properties of proton irradiated polyethersulfone (PES)
}

\author{
NILAM SHAH, DOLLY SINGH, SEJAL SHAH, ANJUM QURESHI, N L SINGH ${ }^{*}$ and \\ K P SINGH ${ }^{\dagger}$ \\ Department of Physics, M.S. University of Baroda, Vadodara 390 002, India \\ ${ }^{\dagger}$ Department of Physics, Punjab University, Chandigarh 160 014, India
}

MS received 26 March 2007; revised 26 June 2007

\begin{abstract}
Polyethersulfone (PES) films were irradiated with $3 \mathrm{MeV}$ proton beams in the fluence range $10^{13}$ $10^{15}$ ions $/ \mathrm{cm}^{2}$. The radiation induced changes in microhardness was investigated by a Vickers' microhardness tester in the load range $100-1000 \mathrm{mN}$ and electrical properties in the frequency range $100 \mathrm{~Hz}-1 \mathrm{MHz}$ by an LCR meter. It is observed that microhardness of the film increases significantly as fluence increases up to $10^{14}$ ions $/ \mathrm{cm}^{2}$. The bulk hardness of the films is obtained at a load of $400 \mathrm{mN}$. The increase in hardness may be attributed to the cross linking effect. There is an exponential increase in conductivity with log frequency and the effect of irradiation is significant at higher fluences. The dielectric constant/loss is observed to change significantly due to irradiation. It has been found that dielectric response in both pristine and irradiated samples obey the Universal law and is given by $\varepsilon \propto f^{n-1}$. These results were corroborated with structural changes observed in FTIR spectra of irradiated samples.
\end{abstract}

Keywords. Polyethersulfone; microhardness; conductivity; dielectric response; FTIR.

\section{Introduction}

The modification of polymer properties by energetic ions is a subject of widespread importance due to the increasing use of polymers in radiation environments encountered in nuclear power plants, space-crafts, sterilization irradiators, high energy particle accelerators etc (Woods and Pikaev 1994; Grossman and Gouzman 2003). The deposited energies effectively modify the chemical structures of the polymers resulting in changes to the dielectric, microhardness and thermal stability (Apel et al 2001). The energy transfer from bombarding ions to the target material results in a complex combination of polymer chain scission, covalent bond breaking and crosslinking. The absorption of radiation energy in sufficient amounts will affect the number of charge carriers in insulating materials to an extent as determined by its molecular structure and chemical composition. The dielectric property of the insulating material is dependent on the number and mobility of the charge carriers present in its structure. The best dielectric materials are those which contain a minimum of charge carriers and potential charge carriers which may be formed by the splitting of covalent, atomic or molecular bonds under the influence of the energetic ions. The dielectric response of a material provides in-

*Author for correspondence (singhnl_msu@yahoo.com) formation about the orientational and translational adjustment of mobile charges present in the dielectric medium in response to an applied electric field. The most important property of dielectric materials is its ability to be polarized under the action of the field. The dielectric loss behaviour of polymer films is very important because of their possible applications for insulation, isolation and passivation in micro-electronic circuits (Dang et al 2002). Polyethersulfone (PES) is finding extensive use in electronics due to its excellent dielectric property. There are only a few reports which mention the effects of low and high energy ions on PES. Wang et al (1991) studied the dose rate effects on the electrical properties of several polymers (PES, PEEK, PS, PAN and PSA) implanted by $50 \mathrm{keV}$ atomic and molecular nitrogen ions. They have reported that the electrical conductivity of these polymers shows significant changes with varying dose rates. Bridwell et al (1991) studied the effects of $50 \mathrm{keV}$ ions of He, C, B, Ar and As on PET, PAN, PES and PEEK polymers. They have reported that aliphatic or partially aliphatic polymers such as PET and PAN have lower resistivity than polymers that have more fully aromatic structures such as PEEK and PES. Evelyn et al (1999) irradiated stacks of thin films of PES, PS and PVC by $5 \mathrm{MeV}$ helium ions and studied the radiation induced changes in chemical structure of the polymers. They observed a complex reorganization of the chemical structure. It was also reported that the disorder increases in the 
deeper irradiated layers of the polymer films due to the nuclear stopping power which increases in the deeper layer. In the present work, radiation induced changes in microhardness and electrical properties of PES at different fluences have been studied. The changes in these properties of the polymer have been corroborated with structural modifications.

\section{Experimental}

Four pieces of PES $\left[\left(\mathrm{C}_{12} \mathrm{H}_{8} \mathrm{O}_{35} \mathrm{~S}\right)_{n}\right.$; density: $\left.1.41 \mathrm{~g} / \mathrm{cm}^{3}\right]$, each of thickness, $275 \mu \mathrm{m}$ and size, $1.5 \times 1.5 \mathrm{~cm}^{2}$, were cut from a sheet supplied by Gharda Chemicals, India. The samples were irradiated by a $3 \mathrm{MeV}$ proton beam at the Physics Department, Panjab University, Chandigarh, India. The current density of the proton beam was $30 \mathrm{nA} / \mathrm{cm}^{2}$ and the irradiation was carried out at fluences of $10^{13}, 10^{14}, 0.5 \times 10^{15}$ and $10^{15}$ ions $/ \mathrm{cm}^{2}$. The proton beam of diameter, $6 \mathrm{~mm}$, was incident perpendicular to the target. The irradiation was carried out under vacuum at a pressure of $10^{-6}$ Torr. Consistency of data was checked by irradiating another set of films at the above fluences. To study the structural changes, including the alteration in position and intensity of characteristic bands, the FTIR spectra of all samples were recorded in the wave number range 4000-500 $\mathrm{cm}^{-1}$ (Bomem, Canada, model-104) with a resolution of $4 \mathrm{~cm}^{-1}$. Vickers' microhardness indentation was carried out on the surface of pristine and irradiated films at different loads in the range $100-1000 \mathrm{mN}$ at room temperature using a Carl Zeiss Axiotech microscope and its accessories. The electrical properties of all samples were studied after irradiation. The resistance, dielectric loss ( $\tan \delta$ and capacitance measurements were carried out using an LCR meter (Hewlett Packard 4284A) over a frequency range $100 \mathrm{~Hz}-1 \mathrm{MHz}$ at room temperature. The a.c. conductivity was calculated using the relation

$$
\sigma=\left(2 \pi f C_{\mathrm{p}} D t\right) \mathrm{A}^{-1}\left(\Omega^{-1} \mathrm{~cm}^{-1}\right) .
$$

The dielectric constant was calculated using the relation

$$
\varepsilon=\mathrm{C}_{\mathrm{p}} / \mathrm{C}_{\mathrm{o}}
$$

where $C_{\mathrm{p}}$ is capacitance measured using the LCR meter, $f$ the frequency, $D$ the dielectric loss and $\varepsilon=\varepsilon_{0} A / t$, where $\varepsilon_{\mathrm{o}}$ is the permittivity of vacuum and $A$ and $t$ are the crosssectional area and thickness of the sample.

\section{Results and discussion}

The electronic $(\mathrm{d} E / \mathrm{d} x)_{\mathrm{e}}$ and nuclear $(\mathrm{d} E / \mathrm{d} x)_{\mathrm{n}}$ stopping power profiles for $3 \mathrm{MeV}$ ions transiting polymer material show that $(\mathrm{d} E / \mathrm{d} x)_{\mathrm{e}}$ dominates at shallower penetration depths compared with $(\mathrm{d} E / \mathrm{d} x)_{\mathrm{n}}$ which is maximum at the end of the ion track. The projected range and the electronic and nuclear stopping powers were calculated using SRIM2000 (Ziegler 2000) code. The projected range of a $3 \mathrm{MeV}$ proton beam in PES was calculated to be $115 \mu \mathrm{m}$, which is 2.4 times less than the thickness of PES film. The electronic stopping power $(\mathrm{d} E / \mathrm{d} X)_{\mathrm{e}}$ and nuclear stopping power $(\mathrm{d} E / \mathrm{d} X)_{n}$ were found to be $1.07 \times 10^{-1} \mathrm{eV} / \AA$ and $6 \cdot 371 \times 10^{-5} \mathrm{eV} / \AA$, respectively.

\subsection{FTIR analysis}

Figure 1 shows the spectra of pristine and irradiated PES samples. The absorption bands as obtained from the pristine spectrum are identified as (A) $720 \mathrm{~cm}^{-1}: \mathrm{CH}_{2}$ absorption bands, (B) $890 \mathrm{~cm}^{-1}: \mathrm{C}-\mathrm{H}$ bending vibration, (C) $1080 \mathrm{~cm}^{-1}: \mathrm{C}=\mathrm{S}$ stretching vibration, (D) $1730 \mathrm{~cm}^{-1}: \mathrm{C}=\mathrm{O}$ stretching vibration, $(\mathrm{E}) 1950 \mathrm{~cm}^{-1}: \mathrm{C}=\mathrm{C}$ stretching vibration and (F) $3000 \mathrm{~cm}^{-1}$ : $\mathrm{C}-\mathrm{H}$ stretching vibration. It is observed that there is no change in overall structure of the polymer but a minor change in intensities was observed up to the fluence of $10^{14}$ ions $/ \mathrm{cm}^{2}$. It may be concluded that PES is resistant to radiation atleast up to the fluence of $10^{14}$ ions $/ \mathrm{cm}^{2}$. The spectrum corresponding to the fluence of $10^{15}$ ions $/ \mathrm{cm}^{2}$ indicates a very significant change in the structure of polymer. This might be due to the breakage of bonds in the structure as well as formation of unsaturated structure. Further at this fluence, the surface became dark brown as seen with naked eyes and surface roughness increased (as observed under optical microscope). This may also contribute in reduction of transmitted intensity.

\subsection{Vickers' microhardness}

For microhardness tests, the indenter employed was a Vickers' pyramidal diamond indenter supplied with the

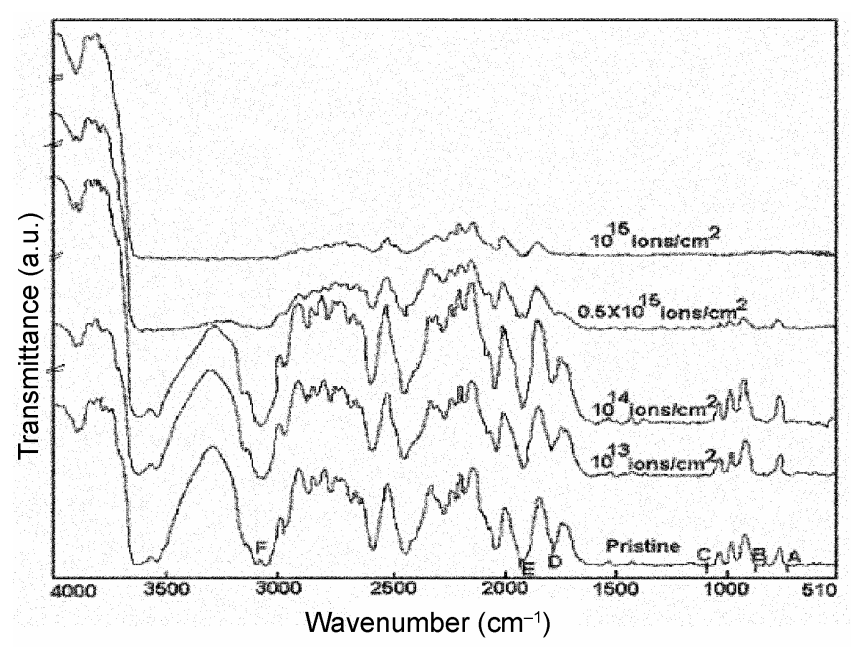

Figure 1. FTIR spectra of pristine and irradiated polyethersulfone films. 
microhardness testing accessory of a Carl Zeiss optical microscope. The microhardness indentations were carried out on the surface of the pristine and irradiated films at room temperature under different applied loads from $100-1000 \mathrm{mN}$ and a constant loading time of $30 \mathrm{~s}$. The Vickers' diamond pyramidal hardness, $H_{\mathrm{v}}$, is the quotient of the applied load, $P$ and the pyramidal surface area, $d^{2}$, of the impression and is given by

$$
H_{\mathrm{v}}=\frac{1 \cdot 854 \times P}{d^{2}}
$$

where $P$ is the applied load in $\mathrm{mN}$ obtained as the product of load in $g$ and gravitational acceleration $\left(g=9.8 \mathrm{~m} / \mathrm{s}^{2}\right), d$ the average of the two indentation marks having diagonal length in $\mu \mathrm{m}$ and $H_{\mathrm{v}}=$ Vickers' hardness in MPa. Figure 2 shows the plot of Vickers' microhardness $\left(H_{\mathrm{v}}\right)$ vs applied load $(P)$ for pristine and irradiated films. It is evident from the figure that the $H_{\mathrm{v}}$ value increases with load up to $200 \mathrm{mN}$, and then decreases. It shows saturation beyond a load of $400 \mathrm{mN}$. The increase of $H_{\mathrm{v}}$ with load can be explained on the basis of the strain-hardening phenomenon. On applying the load, the polymer is subjected to some strain hardening and beyond certain loads the polymer exhausts its strain hardening capacity and the hardness tends to become constant. The rate of strain hardening is greater at low loads and decreases at higher loads (Singh et al 2004a). As can be seen, the hardness becomes independent of the loads for loads more than $400 \mathrm{mN}$. The value obtained from the saturation region, therefore, represents the true hardness of the bulk materials. Since at high loads the indenter penetration depth is also high and surface effects become insignificant. It is also observed that the hardness increases as fluence increases. This may be attributed to the cross-linking phenomenon (Lee et al 1997). At the fluence of $10^{15}$ ions $/ \mathrm{cm}^{2}$, the indentation marks were not seen because of changes in colour of

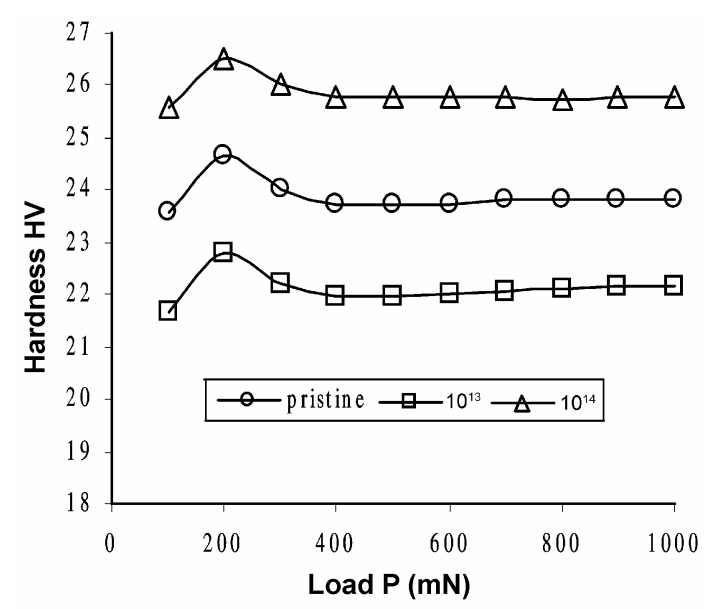

Figure 2. The plots of Vickers' hardness vs applied load for pristine and irradiated polyethersulfone films. sample. It became dark brown in colour. Hence, hardness could not be measured at this fluence.

\subsection{A.C. conductivity}

A.C. conductivity measurement was performed for pristine and irradiated PES samples and is shown in figure 3. A sharp increase in conductivity has been observed in pristine as well as irradiated samples. It is also observed that conductivity increases as fluence increases. The increase in conductivity at a given frequency due to irradiation may be attributed to scissioning of polymer chains, resulting in an increase of free radicals, unsaturation, etc. An a.c. field of sufficiently high frequency may cause a net

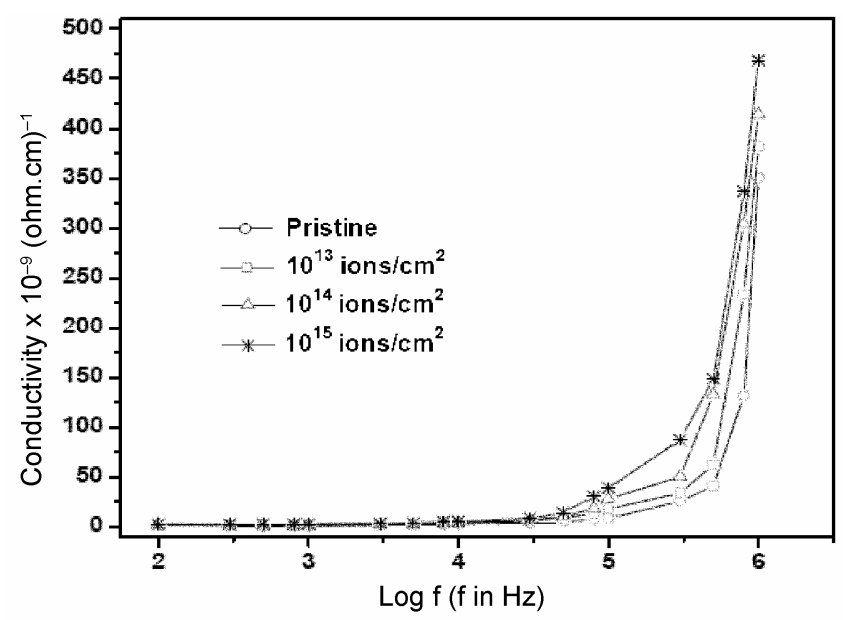

Figure 3. A.C. conductivity vs log frequency plot for pristine and irradiated polyethersulfone films.

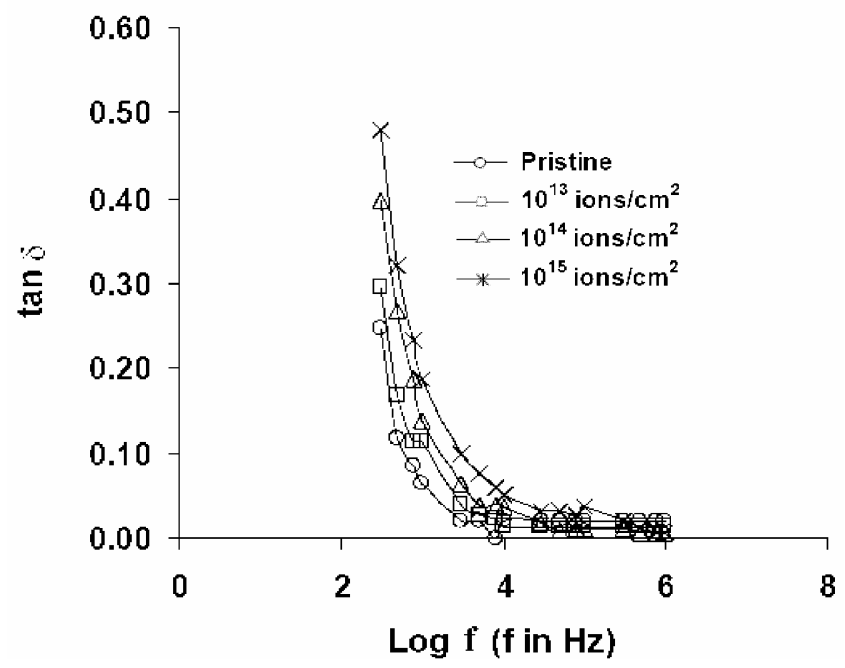

Figure 4. Variation of $\tan \delta$ with $\log$ frequency for pristine and irradiated polyethersulfone films. 


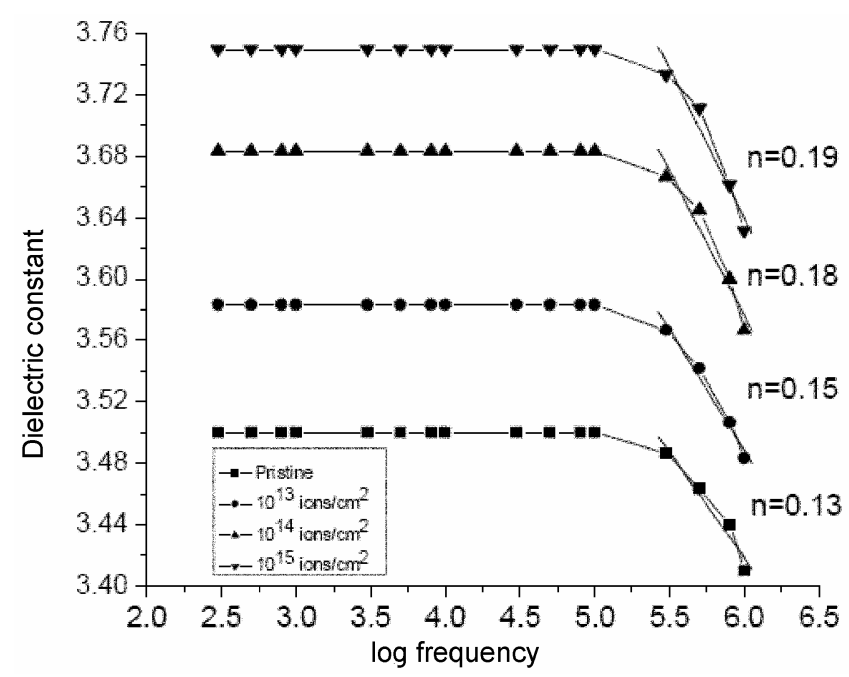

Figure 5. Plot of dielectric constant vs log frequency for pristine and irradiated polyethersulfone films.

polarization, which is out of phase with the field. This results in a.c. conductivity and it appears at frequencies greater than that at which traps are filled or emptied (Jonscher 1977; Singh et al 2004b). Figure 4 shows a plot of $\tan \delta$ (dissipation factor) vs $\log$ frequency for pristine and irradiated PES films. It is observed that loss factor decreases as frequency increases. It is also observed that the loss factor increases moderately with fluence. The increase in loss factor with fluence may be due to scissioning of polymer chains, resulting in an increase of free radicals, unsaturation, etc. Figure 5 shows a plot of dielectric constant $(\varepsilon)$ vs $\log$ frequency at ambient temperature for pristine and irradiated samples. As evident from the graph, the dielectric constant remains almost constant up to $100 \mathrm{kHz}$ and then decreases at higher frequencies. At lower frequency, the mobility of the free charge carriers is constant and thus the dielectric constant is constant. As the frequency increases the charge carriers migrate through the dielectric and get trapped against a defect site and induce an opposite charge in its vicinity. At these frequencies, the polarization of trapped and bound charges cannot take place and hence the dielectric constant decreases. The decrease in dielectric constant at higher frequency can also be explained by Jonscher's power law i.e. $\varepsilon \propto f^{n-1}$, where $n$ varies between zero to one $(0<n<1)$. It is observed that the value of $n$ increases from $0 \cdot 13-$ $0 \cdot 19$ on increasing the fluence (Singh et al 2007).

\section{Conclusions}

The FTIR spectra revealed that PES is a structurally radiation resistant polymer. The minor change in the intensity of functional groups of irradiated samples may be due to breakage of few bands in the ladder structure, but this will not change the overall structure of the polymer. The Vickers' microhardness of the polymer increases as fluence increases and this might be attributed to hydrogen depleted carbon network which make the polymer harder. The increase in dielectric properties due to irradiation may be attributed to scissioning of polymer chains, resulting in an increase of free radicals, unsaturation etc.

\section{Acknowledgements}

The authors are thankful to the operating staff of Cyclotron, Department of Physics, Panjab University, Chandigarh, and Prof. S K Arora, Physics Department, S.P. University, Vallabh Vidyanagar, for providing laboratory facilities. Partial financial support by NSC, New Delhi, is gratefully acknowledged.

\section{References}

Apel P Y, Blonskaya I V, Ogancssian V R, Orelovich O L and Traitman C 2001 Nucl. Instrum. Meth. B185 216

Bridwell L B, Giedd R E, Wang Y Q, Mohite S S, Jahnke T, Brown I M, Bedell C J and Sofield C J 1991 Nucl. Instrum. Meth. B56-57 656

Dang Z M, Shen Y and Nan C W 2002 Appl. Phys. Lett. 814814

Evelyn A L et al 1999 Nucl. Instrum. Meth. B148 1141

Grossman E and Gouzman I 2003 Nucl. Instrum. Meth. B208 48

Jonscher A K 1977 Nature 267673

Lee E H, Rao G R and Mansur L K 1997 Mater. Sci. Forum 248-249 135

Singh N L, Shah N, Desai C F, Singh K P and Arora S K 2004a Rad. Eff. Defects Sol. 159475

Singh N L, Sharma A, Shrinet V, Rakshit A K and Avasthi D K 2004b Bull. Mater. Sci. 27263

Singh N L, Qureshi A, Singh F and Avasthi D K 2007 Mater. Sci. Eng. A457 195

Wang Y Q, Bridwell L B, Giedd R E and Marphy M J 1991 Nucl. Instrum. Meth. B56/57 660

Woods R J and Pikaev A K 1994 Applied radiation chemistry: Radiation processing (New York: John Wiley \& Sons) p. 341

Ziegler J F 2000 SRIM-2000 code, The stopping range of ions in matter (New York: IBM Research) pp 1-28 